\title{
Les effets biologiques des microondes
}

\author{
M. DARDALHON*
}

\begin{abstract}
RÉSUME
La présence et l'utilisation croissantes des ondes électromagnétiques non ionisantes telles que les microondes dans notre environnement, pose le problème de la protection de l'individu mis en présence de ce type de rayonnement. Le présent article fait le point sur les effets biologiques de ces ondes à faible niveau de puissance. Après avoir examiné les résultats obtenus aux niveaux cellulaire, tissulaire ou pluricellulaire, il ressort que les effets décrits sont généralement de petite amplitude, souvent réversibles et il ne semble pas que des changements structuraux importants interviennent. L'absorption non uniforme de l'énergie dans les systèmes biologiques complexes pourrait expliquer certains des effets observés. L'extrapolation des résultats obtenus sur une espèce animale à l'homme reste délicate, car la profondeur de pénétration de l'énergie des microondes varie avec la nature des tissus et la longueur d'onde du rayonnement.
\end{abstract}

\section{ABSTRACT}

The increasing presence and use of non-ionizing electromagnetic waves like microwaves in our environment, raises the problem of the protection of man against this type of radiation. The present paper reviews the biological effects of these waves at low power levels. After an analysis of results obtained at the cellular, tissular or pluricellular levels, it appears that the effects are usually of small amplitude and often reversible. Important structural changes do not seem to occur. The non-uniform absorption of energy in complex biological systems may explain some of the observed effects. The extrapolation of the results obtained in animal species to man remains difficult because the penetration of microwave energy varies as a function of the tissu and the wavelength of the radiation.

\section{INTRODUCTION}

Les rayonnements électromagnétiques non ionisants, tels que les microondes, utilisés d'abord uniquement pour la transmission d'informations ou la détection à distance d'obstacles reçoivent maintenant de nombreuses applications, industrielles, médicales ou même individuelles, qui rendent très important le problème de la protection de l'individu mis

* Institut Curie, Section de Biologie, 26, rue d'Ulm, 75231 Paris Cedex 05. 
en présence de ces ondes. C'est pourquoi de très nombreuses études expérimentales ont été réalisées à différents niveaux d'organisation (moléculaire, cellulaire ou pluricellulaire) des êtres vivants en vue d'aboutir à la détermination des mécanismes d'interaction des microondes avec le matériel biologique. Les résultats de ces études servent généralement de base à l'établissement des normes de sécurité.

Les travaux réalisés, en utilisant de forts niveaux de puissance, ont permis de mettre en évidence les effets thermiques des microondes. En revanche, les interactions des microondes à faible niveau de puissance avec les systèmes biologiques restent plus difficiles à établir. En effet, l'énergie d'activation de ces ondes est trop faible pour produire des ionisations ou des ruptures de liaisons covalentes et les effets biologiques possibles ne peuvent être que de faible amplitude.

II faut noter que dans ce type d'étude, une bonne connaissance des paramètres physiques qui caractérisent la propagation et l'absorption des microondes dans un échantillon biologique revêt un caractère essentiel. La puissance absorbée par un échantillon biologique dans un "champ microonde" est dépendante d'un grand nombre de variables telles que la taille, la configuration et l'orientation de l'objet, la composition de l'échantillon et la fréquence (voir l'article d'A.J. Berteaud p. 7). A cause de ces facteurs, les résultats obtenus avec des échantillons in vitro ou des petits animaux sont difficiles à extrapoler à l'homme. Les résultats sont donc plus exploitables s'ils sont reliés à une énergie absorbée, ce qui peut se faire par des analyses calorimétriques standardisées des échantillons in vitro [50]. Ces analyses aboutissent à la détermination du taux d'absorption spécifique (TAS).

Le but de cet article n'est pas de répertorier tous les travaux concernant l'action des microondes sur les systèmes biologiques, mais de réunir quelques données principales ressortant d'études assez récentes. Nous examinerons donc successivement les résultats obtenus sur les systèmes cellulaires, les tissus ou les systèmes pluricellulaires.

\section{ETUDE DE L'ACTION DES MICROONDES AU NIVEAU CELLULAIRE OU TISSULAIRE}

Les efforts principaux se sont portés vers l'étude de l'interaction des microondes avec les structures et les fonctions cellulaires dans le but d'élucider le mécanisme d'action de ces ondes sur le matériel vivant. De nombreuses études ont été réalisées afin d'examiner si les microondes à faible niveau de puissance pouvaient avoir une action sur le matériel génétique, l'activité enzymatique, les mitochondries, les membranes ou le cytoplasme. La possibilité que les microondes puissent augmenter l'action d'autres agents connus pour altérer le matériel génétique (ADN) a aussi été examinée. Certaines études ont été consacrées à la recherche d'un effet spécifique de la fréquence dans le domaine des ondes millimétriques. Par ailleurs, la recherche d'un effet spécifique d'origine thermique a été motivée par l'utilisation croissante de l'hyperthermie localisée produite par les microondes dans la thérapie des tumeurs cancéreuses. 


\section{1) Action des microondes au niveau du matériel génétique}

De nombreux laboratoires ont étudié l'activité génétique des microondes, à faible niveau de puissance. Dans ce but, ils ont analysé l'apparition de mutations en utilisant des microorganismes ou des cellules de mammifère. Les travaux de Dardalhon et al. [20 - 23] ont été réalisés sur des souches de bactéries ou de levures hautement déficientes pour la réparation des lésions affectant leur matériel génétique. Ces cellules ont été traitées à diverses fréquences de microondes $(9,4,17$ ou $70-75 \mathrm{GHz})$ avec des taux d'absorption spécifique inférieurs à $28 \mathrm{~mW} / \mathrm{g}$. Dans ces conditions, aucun effet significatif n'a été obtenu ni sur la survie, ni sur l'apparition de mutations. Ces résultats sont en accord avec ceux publiés par Blackman et al. [12].

Ces auteurs n'ont observé aucune activité mutagène des microondes en utilisant une souche permettant de détecter les mutations "forward"1 et "reverse" et des expositions à $2,45 \mathrm{GHz}(15$ ou $70 \mathrm{~W} / \mathrm{kg})$ et à $1,70 \mathrm{GHz}$ (3 W/kg).

De même, Dutta et al. [29] ont utilisé la souche D4 de S. cerevisiae permettant de détecter la recombinaison mitotique intragénique (conversion génétique ${ }^{2}$ ) et les souches TA-1535, TA 100 et TA 98 de Salmonella typhimurium permettant de détecter les mutations dues à des substitutions de bases ou à des altérations "frame shift" 3 . En exposant les cellules pendant au moins une génération à des fréquences de $2,45 \mathrm{GHz}$ et 8,5 à $9,6 \mathrm{GHz}$ (pulsé), avec des taux d'absorption voisins de $40 \mathrm{~W} / \mathrm{kg}$, aucun changement génétique n'a été détecté par ces systèmes microbiens très sensibles.

Des résultats récents allant dans le même sens ont été obtenus en utilisant des cellules de mammifères. Ainsi, Lin et Peterson [55] n'ont observé aucune action d'un traitement à $2,45 \mathrm{GHz}(\mathrm{CW})^{4}$ avec 10 à $500 \mathrm{~mW} / \mathrm{cm}^{2}$ sur la viabilité et la croissance de cultures de fibroblastes et lymphoblastes humains. McRee et al. [59] et Galvin et al. [35] n'ont pas observé d'action d'un traitement à $2,45 \mathrm{GHz}(1$ à $100 \mathrm{~mW} / \mathrm{g}$ ) sur l'ultrastructure de cellules myocardiques de l'embryon de caille japonaise de 9 jours. De même, Huang et al. [46] se sont proposés de déterminer si un traitement à 2,45 GHz CW $\left(15 \mathrm{~min} / \mathrm{j}, 5 \mathrm{j}, 0\right.$ à $\left.45 \mathrm{~mW} / \mathrm{cm}^{2}\right)$ pouvait causer des aberrations chromosomiques, des lésions de l'ADN ou d'autres anomalies cellulaires dans des cellules de hamster chinois traitées in vivo dans l'animal. Les résultats montrent qu'aucune différence n'est observée entre les cellules provenant d'animaux traités ou témoins.

En conclusion, on peut noter qu'il existe une grande concordance entre les résultats obtenus sur ce sujet. En effet, en utilisant des systèmes cellulaires divers, une gamme de fréquences assez étendue et des niveaux de puissance faibles ne permettant pas ou très peu d'échauffement

1. Forward: Passage d'un ètat proficient à un état déficient. Reverse : Retour d'un ètat déficient vers un état proficient.

2. Changement génétique non réciproque.

3. Changement dans la lecture des bases de l'ADN.

4. CW: ondes continues. 
des échantillons biologiques, aucune action des microondes au niveau de l'ADN n'a été obtenue.

\section{2) Action des microondes au niveau enzymatique et sur l'activité respiratoire des mitochondries}

La conformation tridimensionnelle d'une enzyme, due à des ponts hydrogènes, est relativement vulnérable. L'énergie nécessaire pour rompre ces ponts est faible. C'est pourquoi de nombreuses équipes de recherche se sont intéressées à l'étude d'un effet éventuel des microondes sur l'activité enzymatique. Ward et al. [84] par exemple, n'ont trouvé aucune différence entre l'activité spécifique à $25^{\circ} \mathrm{C}$ de préparations témoins ou traitées à $2450 \mathrm{MHz}$ et $42 \mathrm{~W} / \mathrm{kg}$ et analysées simultanément par spectrophotométrie et ceci pour diverses préparations telles que la glucose 6 phosphate deshydrogénase, l'adénylate kinase, et la NADPH cytochrome $\mathrm{C}$ réductase. En appliquant cette même technique à la mesure d'activité d'enzymes liées à la membrane, Allis et Fromme [5] n'ont pas observé de différences significatives entre les activités enzymatiques des témoins et des préparations traitées à $2450 \mathrm{MHz}$ (cytochrome oxydase, ATPase, pyruvate kinase, lactate deshydrogénase) et ceci quelle que soit la fréquence de modulation $(16,30,90,120 \mathrm{~Hz})$ utilisée pour un TAS de $26 \mathrm{~mW} / \mathrm{g}$. De la même façon, McRee et al. [59] n'ont pas observé de différences significatives dans l'activité enzymatique de la lactate deshydrogénase, de la glutamique oxaloacétique transaminase et de la créatine phosphokinase isolées de cœurs de caille japonaise traités à $2450 \mathrm{MHz}, 5$ à $20 \mathrm{~mW} / \mathrm{cm}^{2}$ pendant les 8 premiers jours du développement.

Dans l'ensemble, les résultats obtenus sont assez homogènes. Lorsque l'on se situe en dehors des effets thermiques, dans une zone de TAS assez faible (20 à $40 \mathrm{~mW} / \mathrm{g}$ ), aucune action des microondes au niveau enzymatique n'a été observée. En ce qui concerne les mitochondries, Elder et al. [30] n'ont observé aucune différence dans la respiration et la phosphorylation oxydative de mitochondries de foie de rat témoin ou traitées $10 \mathrm{~min}$ à $2,45-3,0$ ou $3,4 \mathrm{GHz}$ à $41 \mathrm{~W} / \mathrm{kg}$. Par contre, Sanders et al. [67] ont exposé des cerveaux de rats à $591 \mathrm{MHz}$ (CW) $\left(5,0\right.$ ou $\left.13,8 \mathrm{~mW} / \mathrm{cm}^{2}\right)$ et ont observé des changements dans les taux de nicotinamide adénine dinucléotide, d'adénosine triphosphate et de créatine phosphate.

\section{3) Action des microondes au niveau membranaire ou cytoplasmique}

En régulant les échanges entre les compartiments intérieurs de la cellule et l'extérieur, les membranes jouent un rôle essentiel. Elles séparent des régions de propriétés diélectriques différentes si bien que des interfaces chargés se forment. Lorsqu'elles sont soumises à un champ électromagnétique, elles peuvent présenter un phénomène de relaxation' qui se produit entre quelques dizaines de kilohertz et quelques centaines de megahertz. Tyazhelov et al. [82] décrivent l'action des microondes à $0,9 \mathrm{GHz}(25$ à $250 \mathrm{~W} / \mathrm{kg})$ sur la conductivité de bicouches phospholipidiques modifiées par de la gramicidine. Ils montrent

1. Lorsqu'une molécule ne peut plus suivre le champ électromagnétique appliqué. 
que les modifications de la conductivité sont dues à la force ionique totale plutôt qu'à la concentration des ions transportés. L'effet observé est dû, selon eux, à un microéchauffement.

D'autres résultats pourraient s'expliquer par un effet des microondes au niveau membranaire. II s'agit des travaux récents concernant la libération d'ions $\mathrm{Ca}^{++}$à partir de tissu cérébral.

Bawin et al. [7] et Blackman et al. [13] ont décrit des augmentations du rapport efflux/influx du calcium dans le tissu cérébral de poulet après 20 min d'exposition à $147 \mathrm{MHz}$. Cet effet ne se produit que pour certaines densités de puissance et certaines fréquences de modulation. C'est ce que l'on appelle un effet fenêtre. De même, les travaux d'Albert et al. [4] montrent qu'un rayonnement électromagnétique de $147 \mathrm{MHz}$, modulation d'amplitude de $16 \mathrm{~Hz}$, provoque une augmentation de $11 \%$ du rapport efflux/influx de calcium dans le tissu pancréatique sans effectuer la libération de protéines marquées dépendantes du calcium.

Ces résultats sont en faveur de l'existence d'une action des microondes à faible niveau de puissance au niveau membranaire. L'équilibre électrochimique entre ions, macromolécules et glycoprotéines de la surface cellulaire pourrait être modifié par de petites variations de la concentration ionique environnante ou des gradients locaux électriques. Par ailleurs, d'autres travaux ont déjà bien établi les lésions membranaires dues aux effets thermiques des microondes.

Les travaux de Motzkin et al. [62] montrent, par exemple, que si l'on traite des suspensions mitochondriales à $25^{\circ} \mathrm{C}$, à $35 \mathrm{GHz}$ et $60-50 \mathrm{GHz}$ pour les densités de puissance supérieures à $100 \mathrm{~mW} / \mathrm{cm}^{2}$, on observe des modifications de la pénétration $\mathrm{du}^{45} \mathrm{Ca}^{++}$pendant le traitement et de la perte de ${ }^{45} \mathrm{Ca}^{++}$après l'arrêt des ondes qui sont dues aux dommages infligés aux membranes par les effets thermiques des microondes.

De même, les travaux de McRee et al. [59], réalisés sur du tissu cardiaque de caille japonaise à $2450 \mathrm{MHz}$, montrent qu'un TAS aussi faible que $10 \mathrm{~mW} / \mathrm{g}$ augmente la perméabilité des cellules cardiaques au bleu de Trypan. A $100 \mathrm{~mW} / \mathrm{g}$, le bleu n'est plus évacué par les cellules.

\section{4) Interaction des microondes avec d'autres agents physiques ou chimiques}

La possibilité que les microondes puissent augmenter les effets d'agents physiques ou chimiques capables d'endommager l'ADN a été examinée.

D'après Dardalhon et al. [22] l'action d'un traitement combiné des microondes $\left(17 \mathrm{GHz}, 50 \mathrm{~mW} / \mathrm{cm}^{2}\right.$ ) et des rayons ultraviolets ou des rayons $\mathrm{X}$ se traduit par une petite augmentation $(0,8$ à $1,65 \%)$ du pourcentage de la recombinaison mitotique. Ceci suggère l'existence d'un effet faible mais réel des microondes qui doit se localiser au niveau du cytoplasme ou des membranes plutôt qu'au niveau de l'ADN. On ne peut, cependant, pas exclure que cet effet soit d'origine microthermique propre aux microondes. Par ailleurs, le groupe de Chang et al. [15] a étudié les effets d'un traitement combiné du Methotrexate (MTX) avec les microondes $\left(1,0 \mathrm{GHz}, 5\right.$ à $\left.50 \mathrm{~mW} / \mathrm{cm}^{2}\right)$. Ils ont observé que ce traitement augmente l'inhibition de la synthèse d'ADN dans les cellules de leucémie murine 
L1210 in vitro. De même, ce traitement prolonge la survie des souris porteuses de L1210 par rapport à celles traitées avec le MTX seul. Cet effet, dont le mécanisme de base n'est pas encore clair, n'a été observé que pour une gamme précise de densités de puissance. C'est ce que l'on appelle un "effet fenêtre".

\section{5) Recherche d'une action spécifique de la fréquence}

D'après des considérations théoriques, Fröhlich [32] a suggéré que de fortes oscillations dans les macromolécules pourraient jouer un rôle de base dans les systèmes biologiques lorsqu'elles se produisent dans une gamme de fréquences allant de 100 à $1000 \mathrm{GHz}$. C'est pourquoi, de nombreuses études ont été réalisées dans la gamme des ondes millimétriques, afin de rechercher l'existence d'effets non thermiques, spécifiques de la fréquence. Les travaux les plus récents dans ce secteur ont été réunis dans un livre publié par Fröhlich et Kremer [33]. Par opposition aux résultats négatifs obtenus par plusieurs équipes [11, 37, 62, 64], des effets positifs ont été décrits par d'autres [10, 39, 40, 41, 42, 86]. L'amplitude des effets observés est toujours très petite $( \pm 10 \%)$. La recherche de ces effets spécifiques est donc très délicate. Elle nécessite l'emploi d'un dispositif permettant d'obtenir une grande stabilité en fréquence, et l'utilisation de tests biologiques très sensibles. Grundler et al. [42] travaillent très activement sur ce sujet. Ils observent des augmentations et des diminutions de la croissance cellulaire en déplaçant la fréquence de $0,01 \mathrm{GHz}$ aux environs de $42 \mathrm{GHz}$.

\section{6) Etude de l'effet thermique des microondes}

La formation des zygotes chez la levure est très sensible à la température, cette propriété a donc été utilisée [20] pour déterminer l'augmentation globale de température d'un système cellulaire après différents traitements aux microondes pour des niveaux de puissance s'étageant de 1 à $60 \mathrm{~mW} / \mathrm{cm}^{2}(10$ à $30 \mathrm{~mW} / \mathrm{g})$. Les résultats obtenus montrent qu'une densité de puissance de $10 \mathrm{~mW} / \mathrm{cm}^{2}$ est équivalente à un échauffement global des cellules de 0,5 à 1 degré suivant la fréquence considérée $(9,4,17$ ou $73 \mathrm{GHz})$. Pour une même puissance, l'échauffement maximal est obtenu pour $17 \mathrm{GHz}$, ce qui suggère qu'il est essentiellement dû à l'absorption diélectrique des ondes par eau libre ou peu liée présente dans le cytoplasme.

En dehors de cet aspect de recherche d'un équivalent thermique des microondes, d'autres études concernant la recherche d'un effet thermique spécifique des microondes se développent dans le but d'optimiser les effets thérapeutiques de l'hyperthermie produite par microondes. Certains travaux réalisés dans ce secteur $[38,78,79]$ sont nettement en faveur de l'existence d'un effet spécifique en plus de l'effet thermique des microondes. Par opposition, d'autres études [24, 45, 56, 58, 66, 68] ne montrent que pas ou peu de différences entre l'effet biologique de l'hyperthermie classique et celui de l'hyperthermie produite par microondes. 


\section{ETUDE DE L'ACTION DES MICROONDES AU NIVEAU PLURICELLULAIRE}

Les études au niveau pluricellulaire sont très diversifiées. Elles concernent l'immunologie, la reproduction, le développement embryonnaire, l'hématologie, les systèmes endocriniens, le système nerveux et le comportement.

\section{1) Immunologie}

Plusieurs études indiquent que les microondes sont susceptibles d'affecter le système immunitaire de petits animaux tels que la souris. Les travaux de Czerski [18] par exemple, décrivent l'existence d'un effet des microondes sur le nombre de cellules formant les anticorps. Le même type de résultat a été obtenu dans différents laboratoires [27, 54]. D'autres équipes décrivent la présence d'altérations immunologiques et hématopoïétiques chez la souris traitée à $2450 \mathrm{MHz}\left(5-15 \mathrm{~mW} / \mathrm{cm}^{2}\right)$ [47] ou encore l'apparition d'une immunostimulation cellulaire chez la souris traitée aux microondes [49]. Par ailleurs, Huang et al. [46] ont observé un changement transitoire, réversible et dose-dépendant du taux de transformation blastique des lymphocytes non stimulés provenant de hamsters chinois traités à $2450 \mathrm{MHz}\left(0,45 \mathrm{~mW} / \mathrm{cm}^{2}, 15 \mathrm{~min} / \mathrm{j}, 5 \mathrm{j}\right)$. D'autres travaux font état d'une augmentation de la transformation morphologique des lymphocytes chez le rat [73] et chez le hamster [46] mais cette augmentation n'a pas été trouvée sur des cellules in vitro [72] et sur la souris traitée à $2450 \mathrm{MHz}$ ou $424 \mathrm{MHz}[74,76]$. De plus, Wiktor-Jedrzejczak et al. [87, 88] décrivent une augmentation de la proportion des lymphocytes B et Schlagel et al. [69] observent une augmentation du nombre des cellules de la rate portant le récepteur complément. Cet effet est dépendant de l'âge de la souris et des facteurs génétiques propres à la souche.

En conclusion, l'ensemble des résultats obtenus dans ce domaine est en faveur de l'existence d'un effet des microondes sur le système immunitaire. Dans la plupart des expérimentations décrites, les animaux ne subissent pas de stress thermique puisque leur température rectale ne varie pas. On ne peut cependant pas exclure complètement que ces effets soient dus à l'existence de points chauds localisés ou à l'existence d'un stress thermique de très faible amplitude que les animaux peuvent compenser par les mécanismes de thermorégulation. II faut noter que la fréquence la plus utilisée dans ces études est $2450 \mathrm{MHz}$. Cette fréquence pénètre complètement le corps d'une souris alors qu'elle ne permet qu'une pénétration superficielle des tissus chez l'homme. L'extrapolation à l'homme reste donc difficile et d'autres expériences avec d'autres espèces animales et dans d'autres conditions expérimentales sont nécessaires.

\section{2) Reproduction et développement embryonnaire}

La possibilité qu'une exposition aux microondes puisse altérer les cellules sexuelles a été considérée dans de nombreux travaux. Contrairement aux travaux anciens dont les résultats étaient contradictoires, les résultats de Hall et al. [44] montrent qu'il n'y a pas d'influence d'un traitement in vitro à $2,45 \mathrm{GHz}(10$ ou $50 \mathrm{~mW} / \mathrm{g})$ sur la capacité fertilisante 
d'un sperme d'oie. Des résultats comparables ont été décrits par Berman et al. [8]. Ces auteurs n'ont pas observé de mutations dans les cellules germinales ni de perturbations de la reproduction chez le rat traité à $2450 \mathrm{MHz}$ ( $5 \mathrm{~mW} / \mathrm{cm}^{2}, 4 \mathrm{~h} / \mathrm{j}, 1$ à 3 mois). De telles perturbations apparaissent pour des niveaux de puissance élevés $\left(28 \mathrm{~mW} / \mathrm{cm}^{2}\right)$ induisant des élévations de la température des testicules. La possible existence d'une action tératogène des microondes a également été très étudiée. Contrairement à ce qui avait été décrit précédemment [14], aucun effet tératogène n'a été observé chez la drosophile $[19,21]$. En ce qui concerne les mammifères, les travaux récents de Inouye et al. [48] montrent qu'un traitement à $2450 \mathrm{MHz}$ $\left(19 \mathrm{~mW} / \mathrm{cm}^{2}, 3 \mathrm{~h} / \mathrm{j}\right.$, les $2^{\mathbf{e}}$ et $3^{\mathbf{e}}$ jours de gestation) n'a pas d'action sur le développement d'embryons de souris préimplantés. Des résultats allant dans le même sens [9] montrent que le taux de survie des fœtus de souris traités in utero à $2450 \mathrm{MHz}\left(28 \mathrm{~mW} / \mathrm{cm}^{2}, 100 \mathrm{~min} / \mathrm{j}\right.$, du $6^{e}$ au $7^{\mathrm{e}}$ jours de gestation) n'est pas affecté. Par contre, les auteurs enregistrent une chute de poids de $10 \%$ des fœtus. Mais il faut noter que le niveau de puissance $\left(28 \mathrm{~mW} / \mathrm{cm}^{2}\right)$ est nettement supérieur aux normes de sécurité.

\section{3) Sang et système cardiovasculaire}

Divers travaux sont en faveur de l'existence d'une action des microondes sur le système cardiovasculaire. A fort niveau de puissance, Reed et al. [65] observent l'apparition d'une tachycardie induite par l'effet thermique. A faible niveau de puissance, ces mêmes auteurs décrivent l'apparition d'une bradycardie du cœur isolé et traité à $960 \mathrm{MHz}(\mathrm{CW})(1-10 \mathrm{~mW} / \mathrm{g})$. Des résultats allant dans le même sens ont été observés par Servantie et al. [71]. Ce groupe a pu mettre en évidence une diminution de la fréquence cardiaque après exposition de rats blancs en "champ microonde" lointain pour des fréquences de $2,45-3$ ou $9,4 \mathrm{GHz}$ ( 5 à $15 \mathrm{j}, 5 \mathrm{~mW} / \mathrm{cm}^{2}$ ).

Clapman et Cain (16) ont obtenu des résultats négatifs en traitant le cœur isolé de grenouille à 3,0 ou $1,5 \mathrm{GHz}$ pulsé. Les travaux de Galvin et al. [35] vont dans le même sens. Ils montrent qu'un traitement continu à $2,45 \mathrm{GHz}(4-16,2 \mathrm{~mW} / \mathrm{g})$ n'a pas d'effet sur l'ultrastructure des cellules du myocarde. Par ailleurs, aucune action des microondes à $2,45 \mathrm{GHz}$ (30 $\mathrm{min}, 2-10 \mathrm{~mW} / \mathrm{g}$ ) n'a été observée sur le taux de contraction ou sur la force contractile de cœurs isolés de rats [36]. Dans l'ensemble, les résultats obtenus par les divers laboratoires ne sont pas homogènes. II est possible que cela soit dû aux différents types de protocoles expérimentaux utilisés et en particulier au fait que les fréquences sont différentes.

En ce qui concerne les études réalisées sur le sang, les microondes à faible niveau de puissance ne semblent pas altérer la structure ou le matériel génétique des cellules [46] ; par contre, des modifications dans les concentrations de $\mathrm{Na}^{+}, \mathrm{K}^{+}$et $\mathrm{Ca}^{2+}$ du sérum sanguin ont été obtenues après exposition de rats à $2880 \mathrm{MHz}$ [34].

\section{4) Système nerveux central, comportement et barrière hémoencé- phalique}

De très nombreuses études ont été menées afin d'examiner si les microondes à faible niveau de puissance étaient susceptibles d'exercer une action mesurable sur les tissus nerveux excitables, le comportement 
et la barrière hémo-encéphalique $[2,6]$. Les résultats décrits sont souvent en relation avec une modification du comportement thermorégulateur [77]. On peut citer, par exemple, les travaux de Adair et Adams [1] qui montrent une modification de la thermorégulation du singe écureuil après une exposition à $2450 \mathrm{MHz}\left(6-8 \mathrm{~mW} / \mathrm{cm}^{2}\right)$. De même, Smialowicz et al. [75] ont montré qu'un traitement à $2450 \mathrm{MHz}\left(90 \mathrm{~min}, 1 \mathrm{~mW} / \mathrm{cm}^{2}\right)$ altère la réponse d'hypothermie induite chez le rat après injection d'une endotoxine.

Par ailleurs, le cerveau a la propriété d'être séparé du sang par une barrière hémo-encéphalique (BHE) qui joue un rôle important dans les phénomènes de diffusion et de transport de médicaments. Certains travaux décrivent un accroissement de la perméabilité de cette barrière après un traitement aux microondes alors que d'autres équipes n'observent aucun effet $[3,31]$. Ainsi, des résultats négatifs ont été obtenus sur des rats exposés à $2450 \mathrm{MHz}$ à faible niveau de puissance [57, 85]. En revanche, un net changement de perméabilité de la BHE est observé lorsque le traitement aux microondes induit des températures de $42{ }^{\circ} \mathrm{C}$ [57].

En ce qui concerne le comportement conditionné, un effet positif a été obtenu après exposition de rats à $2,8 \mathrm{GHz}\left(10 \mathrm{~mW} / \mathrm{cm}^{2}\right)$ [70]. Pour un même niveau de puissance, l'amplitude des effets est plus grande lorsque les animaux sont exposés dans un champ pulsé au lieu d'un champ continu [81]. De plus, le seuil de puissance pour lequel les effets sur le comportement apparaissent dépend de la fréquence. Ce seuil est de $26 \mathrm{~mW} / \mathrm{cm}^{2}$ pour $5,62 \mathrm{GHz}$ et de $15 \mathrm{~mW} / \mathrm{cm}^{2}$ pour $1,28 \mathrm{GHz}$ [25]. Ce résultat s'explique par le fait que les profondeurs de pénétration et la répartition anatomique de l'énergie sont différentes selon la fréquence.

Certaines études récentes font aussi état d'interactions possibles entre les agents pharmacologiques et les microondes $[53,80]$. On peut citer comme exemple l'étude complète qui a été réalisée par Klein et al. [51]. Ces auteurs ont étudié l'action des microondes $2,45 \mathrm{GHz} \mathrm{CW}, 2$ et $5 \mathrm{~mW} / \mathrm{cm}^{2}$ sur 933 rongeurs. Ils concluent à l'absence d'interaction des microondes avec les stimulants et les convulsifiants étudiés, mais à une possibilité de potentialisation des effets comportementaux d'un tranquilisant et à l'existence d'un effet thermogène. Par contre, ils n'observent pas de modifications de la perméabilité de la BHE. Ces auteurs concluent que, compte tenu de la faible amplitude des effets observés sur le système nerveux et de leur caractère souvent réversible, les microondes utilisées à faible niveau de puissance ne constituent pas un rayonnement à haut risque.

\section{5) Organes des sens}

Des effets sur la vision et l'audition ont été décrits. En ce qui concerne les effets oculaires, les travaux réalisés sur l'animal sont en faveur de l'hypothèse selon laquelle l'induction de cataractes est due à un effet thermique des microondes et n'apparaît que pour des niveaux de puissance nettement supérieurs au seuil de sécurité. L'apparition de cataracte serait dépendante de la durée d'exposition et du niveau de puissance des microondes [52]. Par ailleurs, l'effet "auditif" obtenu avec les microondes pulsées a été très étudié par Guy et al. [43]. L'effet serait induit de la même manière qu'un stimulus acoustique conventionnel et serait dû à la conversion de l'énergie des microondes en énergie acoustique par une expansion thermique transitoire du milieu absorbant. 


\section{6) Taux des triglycérides sériques et système neuroendocrinien}

Les effets des microondes sur le système neuroendocrinien des mammifères ont été examinés dans plusieurs laboratoires. II n'y a pas de doute qu'un traitement aux microondes avec un niveau de puissance suffisant induit un stress thermique chez les animaux : ce stress peut agir sur le système neuroendocrinien et entraîner des modifications de l'équilibre hormonal [17, 61]. Ce type d'effet a également été décrit pour de faibles niveaux de puissance de l'ordre de $10 \mathrm{~mW} / \mathrm{cm}^{2}$ induisant de très petits stress thermiques $[63,83]$. On peut citer comme exemple un effet obtenu sur les fonctions corticotrope et gonadotrope chez le rat après traitement à $3,5 \mathrm{GHz}\left(5 \mathrm{à} 10 \mathrm{~mW} / \mathrm{cm}^{2}, 15 \mathrm{j}\right)$ [60]. Cette étude est en bon accord avec les résultats d'un travail réalisé à $2450 \mathrm{MHz}\left(10 \mathrm{~mW} / \mathrm{cm}^{2}\right.$, $24 \mathrm{~h}$ ) et mettant en évidence une perturbation passagère du taux des androgènes circulants [26]. Par ailleurs, une augmentation du taux des triglycérides sériques a été décrite après exposition de souris à $2450 \mathrm{MHz}$ (3 à $\left.12 \mathrm{~mW} / \mathrm{cm}^{2}, 59 \mathrm{~h}\right)$ [28]. Dans l'ensemble, les effets obtenus sur le système neuroendocrinien à faible niveau de puissance sont de petite amplitude et souvent réversibles. On ne peut pas exclure qu'ils soient dus à l'absorption non uniforme de l'énergie dans les animaux. Comme pour le cas des recherches concernant l'immunologie, la plupart des études ont été menées à $2450 \mathrm{MHz}$, fréquence qui pénètre bien un petit animal mais pas l'homme.

\section{CONCLUSION}

En ce qui concerne les expériences réalisées au niveau cellulaire, il semble acquis qu'un traitement aux microondes à faible niveau de puissance $\left(<10 \mathrm{~mW} / \mathrm{cm}^{2}\right)$ ne s'accompagne pas de la production de lésions du matériel génétique (ADN) conduisant à des effets mutagènes ou létaux. De même, aucun effet au niveau enzymatique in vitro n'a été observé. Par contre, l'existence d'un effet cellulaire de faible amplitude et qui pourrait se situer à un niveau cytoplasmique ou membranaire n'est pas exclu. Ces effets sont généralement qualifiés de spécifiques et non thermiques. On ne peut, cependant, pas exclure dans certains cas l'hypothèse d'une action microthermique propre aux microondes.

Pour les expériences réalisées au niveau pluricellulaire, les effets observés à faible niveau de puissance sont toujours de petite amplitude et souvent réversibles. II ne semble pas que des changements structuraux importants interviennent. On peut noter que dans les travaux décrits précédemment, le niveau de puissance est parfois nettement supérieur au seuil de sécurité. Lorsque le niveau de puissance utilisé est susceptible d'induire un léger effet thermique $\left(>10 \mathrm{~mW} / \mathrm{cm}^{2}\right)$, les résultats sont souvent comparés à ceux obtenus en utilisant un moyen classique de chauffage. Les réponses biologiques obtenues dans les deux cas sont, le plus souvent, différentes qualitativement et quantitativement. Ceci provient du fait que, dans les systèmes complexes comme les petits mammifères, l'énergie des microondes est absorbée de façon non uniforme. L'effet biologique obtenu est de ce fait propre aux microondes. Les effets observés au cours d'irradiation à des niveaux de puissance très petits $\left(<10 \mathrm{~mW} / \mathrm{cm}^{2}\right)$ 
étaient jusqu'à présent qualifiés de non thermiques. Cependant, des travaux récents [51] pourraient fournir une explication de nature énergétique à certains de ces effets. De plus, même lorsque le niveau de puissance est très faible $\left(<10 \mathrm{~mW} / \mathrm{cm}^{2}\right)$ et que les animaux ne subissent pas de stress thermique décelable par une augmentation de la température rectale, il n'est pas exclu que les effets observés puissent provenir soit de petites élévations de la température d'un organe particulier du fait de l'absorption non uniforme de l'énergie, soit d'un apport calorifique de petite amplitude pouvant être éliminé rapidement par la thermorégulation physiologique.

Comme l'ont confirmé les résultats expérimentaux [25], le seuil de puissance nécessaire à l'apparition d'un effet biologique varie pour deux fréquences différentes, car la pénétration et la répartition de l'énergie sont différentes dans les deux cas. II est donc difficile d'extrapoler les résultats d'une fréquence à une autre. De même, l'extrapolation d'une espèce à l'autre est délicate, car l'absorption des microondes varie selon l'organe et selon la taille de l'espèce traitée. De plus, on ne peut pas exclure que certains organes appartenant à des espèces distinctes puissent présenter des sensibilités différentes qui peuvent être déterminées génétiquement. Les normes de sécurité ont été établies en tenant compte des résultats expérimentaux obtenus et plus particulièrement, de ceux obtenus sur le comportement et le système nerveux. Ce dernier constitue une cible relativement sensible du fait de la nature des tissus et des phénomènes de résonance susceptibles de créer des points chauds dans la tête. L'analyse de la plupart des résultats expérimentaux montre qu'ils apparaissent pour des TAS supérieurs à $4 \mathrm{~W} / \mathrm{kg}$ en moyenne sur l'organisme entier pour des durées d'exposition d'environ 1 heure. Les normes de sécurité ont donc été établies nettement en-dessous de cette valeur pour tenir compte, d'une part, des risques d'interaction des microondes avec d'autres agents physiques ou chimiques et, d'autre part, des risques de résonance qui peuvent aboutir à la formation de points chauds, en particulier pour la zone des fréquences comprises entre 30 et $400 \mathrm{MHz}$ pour laquelle on observe l'existence de phénomènes de résonance chez l'homme.

\section{BIBLIOGRAPHIE}

[1] ADAIR E.R., ADAMS B.W. Microwaves modify thermoregulatory behavior in squirrel monkey. Biolectromagnetics, 1980, 1, 1-20.

[2] ADEY W.R. Tissue interactions with non ionizing electromagnetic field. Physiol. Rev., 1981, 61, 435-514.

[3] ALBERT E.N. Current status of microwave effects on the blood brain barrier. J. Microwave Power 1979, 14, 281-285.

[4] ALBERT E., BLACKMAN C., SLABY F. Calcium dependent secretory protein release and calcium efflux during RF irradiation of rat pancreatic tissue slices. In : Symposium international URSI "Ondes électromagnétiques et biologie", Jouy en Josas, Juillet 1980. (A.J. Berteaud et B. Servantie, Eds.). Paris : CNRS, $1981,325-329$

[5] ALLIS J.W. and FROMME, M.L. Activity of membrane-bound enzymes exposed to sinusoidally modulated $2450 \mathrm{MHz}$ microwave radiation. Radioscience, 1979 , $65,85-91$. 
[6] BARANSKI S., EDELWEJN Z. Experimental morphologic and electroencephalographic studies of microwave effects on the nervous system, Ann. N.Y. Acad. Sci., 1975, 247, 109-116.

[7] BAWIN S.M., SHEPPARD A.R., ADEY W.R. Models of long-range order in cerebral macromolecules : effects of ELF, VHF and UHF fields. In : International symposium on the biological effects of electromagnetic waves, 1977 abstracts of scientific papers. Washington : USNC - URSI, 1977, 100.

[8] BERMAN E., CARTER H.B., HOUSE D. Tests of mutagenesis and reproduction in male rats exposed to $2450 \mathrm{MHz}(\mathrm{CW})$ microwaves. Bioelectromagnetics, $1980,1,65-76$.

[9] BERMAN E., CARTER H.B., HOUSE D. Reduced weight in mice offspring after in utero exposure to $2450 \mathrm{MHz}(\mathrm{CW})$ microwaves. Bioelectromagnetics, 1982 , 3. 285-291.

[10] BERTEAUD A.J., DARDALHON M., REBEYROTTE N. et AVERBECK D. Action d'un rayonnement électromagnétique à longueur d'onde millimétrique sur la croissance bactérienne. C.R. Acad. Sc., Paris, 1975, 281, 843-846.

[11] BLACKMAN C.F., BENANE S.G., WEIL C.M. and ALI J.S. Effects of nonionizing electromagnetic radiation on single-cell biologic systems. Ann. N.Y. Acad. Sci., $1975,247,352-366$.

[12] BLACKMAN C.F., SURLES M.C. and BENANE S.G. The effect of microwave exposure on bacteria : mutation induction. In : Biological effects of electromagnetic waves, USNC/URSI, Annual meeting, Boulder, Colorado, Oct. 20-23, 1975, HEW publication $n^{\circ} 77-8010,1978,1$.

[13] BLACKMAN C.F., BENANE S.G., ELDER J.A. HOUSE D.E., LAMPE J.A. and FAULK J.M. Induction of calcium ion efflux from brain tissue by radiofrequency on the power-density window. Bioelectromagnetics, 1980, 1, 35-43.

[14] CARPENTER R.L., LIVSTONE E.M. Evidence for non thermal effects of microwave radiation : abnormal development of irradiated insect pupae. IEEE Trans. Microwave Theory Technol., 1971, 19, 173-178.

[15] CHANG B.K., HUANG A.T. and JOINES W.T. Inhibition of DNA synthesis and enhancement of the uptake and action of methotrexate by low power density microwave radiation in L1210 leukemia cells. Cancer Res., 1980, 40, 1002-1005.

[16] CLAPMAN R.M., CAIN C.A. Absence of heart rate effects in isolated frog heart with pulse modulated microwave energy. J. Microwave Power, 1975, 10, 411-419.

[17] COLLINS K.J. and WEINER J.S. Endocrinological aspects of exposure of high environmental temperatures. Physiol. Rev., 1968, 48, 785.

[18] CZERSKI P. Microwave effects in the blood-forming system with particular reference to the lymphocyte, Ann. N.Y. Acad. Sci., 1975, 247, 232-242.

[19] DARDALHON M., AVERBECK D. Quelques actions des microondes chez les bactéries, les levures et les drosophiles. In : Effets biologiques des rayonnements non ionisants, utilisation et risques associés, Nainville les Roches. 22-26 Mai 1978, S.I : SFRP, 1978, 279-299.

[20] DARDALHON M., AVERBECK D. and BERTEAUD A.J., Determination of a thermal equivalent of millimeter microwaves in living cells, J. Microwave Power, 1979, 14, (4), 307-312.

[21] DARDALHON M., BERTEAUD A.J., AVERBECK D. Microwave effects in drosophila melanogaster. Radioprotection, 1979, 14, 145-159.

[22] DARDALHON M., AVERBECK D., BERTEAUD A.J. Action des ondes centimétriques seules ou combinées avec les rayons ultraviolets sur les cellules eucaryotiques. In : Symposium international URSI "Ondes électromagnétiques et biologie", Jouy en Josas, Juillet 1980 (A.J. Berteaud et B. Servantie, Eds.). Paris : CNRS, 1980, 17-24. 
[23] DARDALHON M., AVERBECK D., BERTEAUD A.J. Studies on possible genetic effects of microwaves in procaryotic and eucaryotic cells. Radiat. Environ. Biophys., 1981, 20, 37-51.

[24] DARDALHON M., MORÉ C., AVERBECK D., BERTEAUD A.J. Thermal action of 2.45 $\mathrm{GHz}$ microwaves on the cytoplasm of chinese hamster cells. Bioelectromagnetics (sous presse).

[25] DE LORGE J.O., EZELL C.S. Observing responses of rats exposed to 1.28 and 5.62 GHz microwave. Bioelectromagnetics, 1980, 1, 183-198.

[26] DESCHAUX P., PELISSIER J.P., SANTINI R., FONTANGES R. Effets d'un rayonnement dû aux microondes sur l'axe hypophysotesticulaire chez le rat blanc. In : Effets biologiques des rayonnements non ionisants, utilisation et risques associés, Nainville les Roches, 22-26 mai 1978, S.I. : SFRP, 1978, 265-268.

[27] DRAUSSIN M., CHEVALIER J.M., LAGOUTTE J.H., SERRE A., GOUJET P., SERVANTIE B., MIRO L., BUREAU J.P. Effets des ondes centimétriques à $3 \mathrm{GHz}$ pulsés sur la réponse immunitaire in vivo chez la souris. In: Symposium international URSI " Ondes électromagnétiques et biologie", Jouy-en-Josas, Juillet 1980 (A.J. Berteaud et B. Servantie, Eds). Paris: CNRS, 1980, 133-137.

[28] DUMAS J.C., NOUGAROLIS D., STOLL M., PLURIEN G. Influence de la durée d'exposition aux microondes sur la triglycéridémie de la souris. In : Symposium international URSI "Ondes électromagnétiques et biologie" Jouy-en-Josas, Juillet 1980, (A.J. Berteaud et B. Servantie, Eds). Paris : CNRS, 1980, 75-77.

[29] DUTTA S.K., NELSON W.H., BLACKMAN C.F. and BRUSICK D.J. Lack of microbial genetic response to $2,45 \mathrm{GHz} C W$ and 8,5 to $9,6 \mathrm{GHz}$ pulsed microwaves, J. Microwave Power, 1979, 14, 275-280.

[30] ELDER J.A. and ALI J.S. The effect of microwaves $(2450 \mathrm{MHz})$ on isolated rat liver mitochondria. Ann. N.Y. Acad. Sci., 1975, 247, 251-262.

[31] FREY A.H. Studies of the blood brain barrier: preliminary findings and discussion, Radio Sci., 1979, 14, (65), 349-350.

[32] FROHLICH H. Long range coherence and the action of enzymes. Nature, 1970, 228, 1093.

[33] FRÖHLICH H. and KREMER F. Coherent excitations in biological systems, Berlin : Springer-Verlag, 1983.

[34] FURMANIAK A. Quantitative changes in potassium, sodium and calcium in the submaxillary salivary gland and blood serum of rats exposed to $2880 \mathrm{MHz}$ microwave radiation. Bioelectromagnetics, 1983, 4, 55-62.

[35] GALVIN M.J., MCREE D.I., LIEBERMAN M. Effects of $2.45 \mathrm{GHz}$ microwave radiation on embryonic quail hearts. Bioelectromagnetics, 1980, 1, 389-396.

[36] GALVIN M.J., DUTTON M.S., McREE D.I. Influence of $2.45 \mathrm{GHz}$ microwave radiation on spontaneously beating rat atria. Bioelectromagnetics, 1982, 3, 219-226.

[37] GANGHI O.P., HAGMANN M.J., HILL D.W., PARTLOW L.M. and BUSH L. Millimeter wave absorption spectra of biological samples. Bioelectromagnetics, $1980,1,285-298$.

[38] GILLOIS M., AUGE C., CHEVALET C. Effet des ondes électromagnétiques non ionisantes sur la viabilité et l'hérédité des cellules de mammifères en culture établie. In : Symposium international URSI "Ondes électromagnétiques et biologie", Jouy-en-Josas, Juillet 1980 (A.J. Berteaud et B. Servantie, Eds.). Paris : CNRS, $1980,9-15$. 
[39] GRINGANZ K.I., I.A. ZHULIN, A.D. SYTINSKII, N.D. DEUYATKOV, E.B. BAZANOVA, A.K. BRYUKHOVA, R.L. VILENSKAYA, E.A. GELVICH, M.B. GOLANT, N.S. LANDAU, V.M. MELNIKOVA, N.P. MIKAELYAN, G.M. OKHOKHONINA, L.A. SEVASTYANOVA, A.Z. SMOLYANSKAVA, N.A. SYCHEVA, R.L. VILENSKAYA, V.F. KONRATEVA, E.N. CHRISTYAKOVA, I.R. SHMAKOVA, N.B. IVANOVA, A.A. TRESKUNOV, S.E. MANOILOV, M.A. STRELKOVA, N.P. ZALYUBOVSKAYA, R.I. KISELEV, N.P. ZALYUBOVSKAYA, V.I. GAIDUK, Y.I. KHURGIN and V.A. KUDRYASHOVA. Scientific session of the division of general physic and astronomy, URSS academy of sciences. Sov. Phys. Usp. (Translation) 1974, 16, (4), 568-569.

[40] GRUNDLER W., KEILMANN F. and FRÖHLICH H. Resonant growth rate response of yeast cells irradiated by weak microwaves. Phys. Lett., 1977, 62 A, 463-466.

[41] GRUNDLER W. and KEILMANN F. Non thermal effects of millimeter microwaves on yeast growth. Z. Naturf. C., 1978, 33, 15-22.

[42] GRUNDLER W., KEILMANN F., PUTTERLIK V., SANTO L., STRUBE D., ZIMMERMANN I. Non thermal resonant effects of $42 \mathrm{GHz}$ microwaves on the growth of yeast cultures. In : Coherent excitations in biological systems (Fröhlich $\mathrm{H}$. and Kremer F., Eds). Berlin : Springer-Verlag, 1983, 21-37.

[43] GUY A.W., CHOU G.K., LIN J.C., CHRISTENSEN D. Microwave-induced acoustic effects in mammalian auditory systems and physical materials. In : Biologic effects of non ionizing radiation (Tyler P.Ed.). Ann. N.Y. Acad. Sci., 1975, 245, 194.

[44] HALL C.A., MCREE D.I., GALVIN M.J., WHITE N.B., THAXTON J.P., CHRISTENVEN V.L. Influence of in vitro microwave radiation on the fertilizing capacity of turkey sperm. Bioelectromagnetics, 1983, 4, 43-54.

[45] HARRISON G.H., ROBINSON J.E., MCCULLOCH D., CHEUNG A.Y. Comparison of hyperthermal cellular survival in the presence or absence of $2,45 \mathrm{GHz}$ microwave radiation. In : Symposium international URSI "Ondes électromagnétiques et biologie", Jouy-en-Josas, Juillet 1980, (A.J. Berteaud et B. Servantie, Eds.). Paris: CNRS, 1980, 41-45.

[46] HUANG A.T., ENGLE M.E., ELDER J.A., KINN J.B. and WARD, T.R. The effect of microwave radiation $(2450 \mathrm{MHz})$ on the morphology and chromosomes of lymphocytes. Radiosci. 1977, 12, (6S), 173-177.

[47] HUANG A., T.F., MOLD N.G. Immunologic and hematopoietic alterations by $2450 \mathrm{MHz}$ electromagnetic radiation. Bioelectromagnetics, 1980, 1, 77-87.

[48] INOUYE M., MATSUMOTO N., GALVIN M.J., McREE D.I. Lack of effects of $2.45 \mathrm{GHz}$ microwave radiation on the development of preimplantation embryos of mice. Bioelectromagnetics, 1982, 3, 275-283.

[49] IVANOFF B., ROBERT D., DESCHAUX P., PELLISSIER J.P., FONTANGES R. Effets des microondes sur la réponse immunitaire cellulaire de la souris Swiss. C.R. Soc. Biol., 1979, 173, 932-936.

[50] JOHNSON C.C., DURNEY C.H., BARBER P.W., MASSOUDI H., ALLEN S.J. and $\mathrm{MITCH}$ J.C. Descriptive summary : Radio-frequency radiation dosimetry handbook. Radiosci., 1977, 12, (6S), 57-59.

[51] KLEIN M.J., STERU L., MILHOUD C.L., ROSOLEN S.G. Application de techniques pharmacologiques à l'étude des effets biologiques des microondes au cours d'irradiation à de faibles niveaux de puissance. Radioprotection, 1982. 17, (4), 225-241.

[52] KRAMER P.O., EMERY A.F., GUY A.W., and LIN J.C. The ocular effects of microwaves on hypothermic rabbits : and study of microwave cataractogenic mechanisms. Ann. N.Y. Acad. Sci., 1975, 247, 155.

[53] LAI H., HORITA A., CHOU C.K., GUY A.W. Psychoactive drug response is affected by acute low level microwave irradiation. Bioelectromagnetics, 1983, 4, 205-214. 
[54] LIDDLE C.G., PUTNAM J.P., ALI J.S., LEWIS J.Y., BELL B., WEST M.W. and LEWTER O.H. Alteration of circulating antibody response of mice exposed to $9 \mathrm{GHz}$ pulsed microwaves. Bioelectromagnetics, 1980, 1, 397, 404.

[55] LIN J.C., PETERSON W.D. Cytological effects of $2450 \mathrm{MHz}$ CW microwave radiation J. Bioengineering, 1977, (1), 471-478.

[56] LIN J.C., OTTENBREIT M.J., WANG S., INOUE S., BOLLINGER R.O., FRACASSA M. Microwave effects on granulocyte and macrophage precursor cells of mice in vitro. Radiat. Res., 1979, 80, 292-302.

[57] LIN J.C., LIN M.F. Studies on microwave and blood-brain barrier interaction. Bioelectromagnetics, 1980, 1, 313-323.

[58] LIVINGSTON G.K., JOHNSON C.C., DETHLEFSEN L.A. Comparative effects of waterbath and microwave induced hyperthermia on survival of chinese hamster ovary (CHO) cells. Radiosci, 1979, 14, (6S), 117-123.

[59] Mac REE D.I., GALVIN M., HALL C. and LIEBERMAN M. Microwave effects on embryonic cardiac tissue of japonese quail. In : Symposium international URSI "Ondes électromagnétiques et biologie", Jouy-en-Josas, juillet 1980 (A.J. Berteaud, Servantie B., Eds.). Paris : CNRS, 1980, 79-84.

[60] MANIEZ J., LE RUZ P., PLURIEN G. Effets de l'exposition néonatale aux microondes sur l'évolution ultérieure des fonctions corticotrope et gonadotrope chez le rat. In : Symposium international URSI, "Ondes électromagnétiques et biologie", Juillet 1980, Jouy-en-Josas, Juillet 1980 (A.J. Berteaud, B. Servantie, Eds). Paris : CNRS, 1980, 85-88.

[61] MICHAELSON S.M., THOMSON R.A.E., QUINLAN W.J. Effect of electromagnetic radiations on physiological responses, Aerosp. Med., 1967, 38, 293-298.

[62] MOTZKIN S.M., MELNICK R.L., RUBENSTEIN C., ROSENTHAL S., BIRENBAUM L. Effets of CW millimeter wave irradiation on mitochondrial oxidative phosphorylation and $\mathrm{Ca}^{++}$transport. In : Symposium international URSI "Ondes électromagnétiques et biologie", Jouy-en-Josas Juillet 1980, (A.J. Berteaud, B. Servantie, Eds). Paris : CNRS, 1980, 109-115.

[63] PARKER L.N. Thyroid suppression and adrenomedullary activation by low intensity microwave radiation. Ann. J. Physiol., 1973, 224, 1388.

[64] PARTLOW L.M., BUSH L.G., STENSAAS L.J., HILL D.W., RIAZI A. and GANDHI O.P. A search for frequency specific bioeffects caused by microwave irradiation, abstract. National radioscience meeting. Bioelectromagnetics symposium, Seattle USA, 1979, BEMS Poster B-7, p. 464.

[65] REED J.R., LORDS J.L., DURNEY C.H. Microwave irradiation of the isolated rat heart after treatment with ANS blocking agents. Radiosci., 1977, 12, (6S) 161-165.

[66] ROBINSON J.E., HARRISON G.H., MCCULLOCH D., MCCREADY W.A., CHEUNG A.Y. The effects of microwaves on cell survival at elevated temperatures. Radiat. Res., $1981,88,542-551$.

[67] SANDERS A.P., SCHAEFFER D.J., JOINES W.T. Microwave effects on energy metabolism of rat brain. Bioelectromagnetics, 1980, 1, 171-181.

[68] SAPARETO S.A., LI G.C., WHITER K.A., HAHN G.M., VAGUINE V.A., GIEBELER R.H., TANABE E. Microwave cytotoxicity : lack of in vitro evidence for non thermal effects at high power levels. Radiat. Res. 1982, 89, 124-133.

[69] SCHLAGEL C.J., SULEK K., HO H.S., LEACH W.M., AHMED A., WOODY J.N. Biologic effects of microwave exposure. II. Studies on the mechanisms controlling susceptibility to microwave induced increase in complement receptor positive spleen cells. Bioelectromagnetics, 1980, 1, 405-414.

[70] SCHROT J., THOMAS J.R., BANVARD R.A. Modification of the repeated acquisition of response sequences in rats by low level microwave exposure. Bioelectromagnetics, 1980, 1, 89-99. 
[71] SERVANTIE B., CRETON B., BRUSCHERA D., ROUSSEL J. Ralentissement de la fréquence cardiaque après exposition à un champ de microondes chez le rat blanc, In : Symposium international URSI "Ondes électromagnétiques et biologie", Jouy-en-Josas, Juillet 1980, (A.J. Berteaud, B. Servantie, Eds). Paris : CNRS, 1980, 67-69.

[72] SMIALOWICZ R.J. The effect of microwaves $(2450 \mathrm{MHz})$ on lymphocyte blast transformation in vitro. Selected paper of the USNC/URSI. Annual meeting Boulder, Colorado, Oct. 1975, HEW publication n 77-8010, 1976, 1, 472-483.

[73] SMIALOWICZ R.J., KINN J.B., ELDER J.A. Perinatal exposure of rats to $2450 \mathrm{MHz}$ CW microwave radiation: effects on lymphocytes. Radiosci., 1979, 14, 147-153.

[74] SMIALOWICZ R.J., RIDDLE M.M., BRUGNOLOTTI P.L., SPERRAZZA J.M. and KINN J.B. Evaluation of lymphocyte function in mice exposed to $2450 \mathrm{MHz}$ (CW) microwaves, In : Symposium on electromagnetic fields in biological systems, Ottawa, Canada, June 1978 (S. Stuchly, Ed.), 1979, 122-152.

[75] SMIALOWICZ R.J., COMPTON K.L., RIDDLE M.M., ROGERS R.R., BRUGNOLOTTI P.L. Microwave radiation $(2450 \mathrm{MHz})$ alters the endotoxin-induced hypothermic response of rats. Bioelectromagnetics 1980, 1, 353-361.

[76] SMIALOWICZ R.J., RIDDLE M.M., WEIL C.M., BRUGNOLOTTI P.L. and KINN J.B. Assessment of the immune responsiveness of mice irradiated with continuous wave or pulse-modulated $425-\mathrm{MHz}$ radio-frequency radiation. Bioelectromagnetics, 1982, 3, 467-470.

[77] STERN S., MARGOLIN L., WEISS B., LU S.T., MICHAELSON S.M. Microwaves : effects on thermoregulatory behaviour in rats. Science, 1979, 206, 1198-1201.

[78] SUBJECK J., SCIANDRA J., REPASKY E., JOHNSON R. An assay system for the study of the athermal effects of microwaves. In : Symposium international URSI " Ondes électromagnétiques et biologie" Jouy-en-Josas, Juillet (A.J. Berteaud, B. Servantie, Eds). Paris: CNRS, 1980, pp. 321-323.

[79] TCHAO Y.H., RADZISZEWSKI E., SAUZIN-MONNOT M.J. Effet cumulatif des microondes. In : Symposium international URSI "Ondes électromagnétiques et biologie ", Juillet 1980, Jouy-en-Josas, (A.J. Berteaud, B. Servantie, Eds). Paris: CNRS, $1980,47-51$.

[80] THOMAS J.R., MAITLAND G. Microwave radiation and dextroamphetamine : evidence of combined effects on behavior of rats. Radiosci., 1978, 14, (65), 253-258.

[81] THOMAS J.R., SCHROT J., BANVARD R.A. Comparative effects of pulsed and continuous wave $2.8 \mathrm{GHz}$ microwaves on temporally defined behavior. Bioelectromagnetics, 1982, 3, 227-235.

[82] TYAZHELOV V.V., ALEKSEEV S.I., FAISOVA L.Kh., CHERTISHCHEV V.V. Peculiarities of microwave effect on gramicidin modified bilayers. In : Symposium international URSI "Ondes électromagnétiques et biologie", Jouy-en-Josas, Juillet 1980 (A.J. Berteaud, B. Servantie, Eds). Paris : CNRS, 1980, 105-108.

[83] WANGEMANN R.T. and CLEARY S.F. The in vivo effects of $2,45 \mathrm{GHz}$ microwave radiation on rabbit serum components. Radiat. Environ. Biophys., 1976, 13, 89-94.

[84] WARD T.R., ALLIS J.W. and ELDER J.A. Measure of enzymatic activity coincident with $2450 \mathrm{MHz}$ microwave exposure. J. Microwave Power, 1975, 10, 315-320.

[85] WARD T.R., ELDER J.A., LONG M.D., SVENDSGAARD D. Measurement of bloodbrain barrier permeation in rats during exposure to $2450 \mathrm{MHz}$ microwaves. Bioelectromagnetics, 1982, 3, 371-383.

[86] WEBB S.J., DODDS D.E. Inhibition of bacterial cell growth by 136 gc microwaves, Nature, $1968,218,374-376$.

[87] WIKTOR-JEDRZEJCZAK W., AHMED A., CZERSKI P., LEACH W.M., SELL K.W. Immune response of mice to $2450 \mathrm{MHz}$ microwave radiation : overview of immunology and empirical studies of lymphoïd splenic cells. Radiosci., 1977, 12, (65) 209-219.

[88] WIKTOR-JEDRZEJCZAK W., AHMED A., CZERSKI P., LEACH W.M. and SELL K.W. Effect of microwaves $(2450 \mathrm{MHz})$ on the immune system in mice : studies of nucleic acid and protein synthesis. Bioelectromagnetics, 1980, 1, 161-170. 